\title{
ISOPERIMETRIC INEQUALITIES INVOLVING HEAT FLOW UNDER LINEAR RADIATION CONDITIONS
}

\author{
ANDREW ACKER
}

\begin{abstract}
Under the assumption that a constant linear radiation condition holds on each boundary component, we show that the annulus and the strip are heat-flow minimizing with respect to area-preserving variations in one (for the annulus: the outer) boundary component.
\end{abstract}

1. Introduction. As in Figure 1 , let $\Omega$ represent a simply connected region relative to $[0,1] \times R$ whose boundary relative to $[0,1] \times R$ consists of two simple arcs $\Gamma^{*}$ and $\Gamma$. (Here, $\Gamma^{*}$ and $\Gamma$ have bounded curvature and are horizontal at their endpoints.) The heat flow crossing $\Omega$ is defined by $H=-\int_{\Gamma^{*}} \mathbf{D}_{n} u(p)|d p|$, where the temperature $u(p)$ is a harmonic function on $\Omega$ which satisfies the boundary conditions: $\mathbf{D}_{n} u=\lambda u$ on $\Gamma, \mathrm{D}_{n} u=\lambda^{*}(u-1)$ on $\Gamma^{*}$, and $D_{x} u=0$ on $(\{0,1\} \times R) \cap \Omega$. (Here $\lambda$ and $\lambda^{*}$ are positive constants and $\mathbf{D}_{n} u(p)$ at $p \in \Gamma^{*} \cup \Gamma$ is the normal derivative directed into s.)

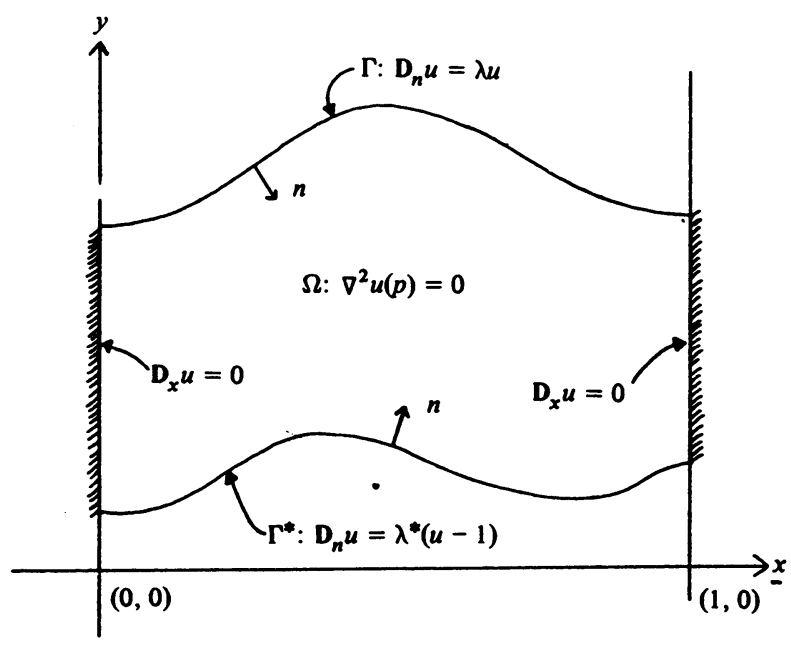

FIGURE 1. Definitions of $\Omega, \Gamma, \Gamma^{*}$ and $u(p)$.

We assume $\Gamma^{*}=[0,1] \times\{0\}$ in $\$ 2$.

Received by the editors August 23, 1976.

AMS (MOS) subject classifications (1970). Primary 35B99; Secondary 35J23, $35 \mathrm{~J} 05$. 
For any fixed $A>0$, let $\{\Omega\}_{A}$ be the class of regions $\Omega$ such that $\Gamma^{*}=[0,1] \times\{0\},|\Omega| \leqslant A$ (where $|\Omega|$ is the area of $\Omega$ ), and the (inwardly directed) normal to $\Gamma$ never points in the positive $y$-direction. In \$2, we show that the region $[0,1] \times(0, A)$ is uniquely heat-flow minimizing in $\{\Omega\}_{A}$. It is intuitive that the horizontal strips [0,1] $\times(\alpha, \alpha+A), \alpha \in R$, should be heatflow minimizing in the class of all regions $\Omega$ having $|\Omega| \leqslant A$. This more general (probable) isoperimetric property of the strips $[0,1] \times(\alpha, \alpha+A)$ has not been proved. In the limiting case where $\lambda=\lambda^{*}=\infty$, the Newton radiation conditions reduce to the Dirichlet conditions $u=1$ on $\Gamma^{*}$ and $u=0$ on $\Gamma$. In this case the general heat-flow-minimizing property of the strips $[0,1]$ $\times(\alpha, \alpha+A)$ is equivalent to the main lemma in the proof of Grötzsch's Principle [3, pp. 140-142]. The analogous heat-flow-minimizing property of the annulus was proven by Carleman [2] and Szegö [7]. For the case where linear radiation conditions apply, a heat-flow-minimizing property of the annulus is discussed in $\$ 3$.

2. The main results. Let $\bar{\Omega}, \bar{\Gamma}, \bar{\Gamma}^{*}, \bar{u}(p)$, and $\bar{H}$ refer to a second heat problem for which the assumptions in $\$ 1$ hold. We assume throughout this section that $\Gamma^{*}=\bar{\Gamma}^{*}=[0,1] \times\{0\}$.

The temperature on $[0,1] \times(0, A)$ (for any $A>0)$ subject to our boundary conditions is

$$
w_{A}(x, y)=w_{A}(y)=\left(\lambda^{*}+\lambda \lambda^{*}(A-y)\right)\left(\lambda+\lambda^{*}+\lambda \lambda^{*} A\right)^{-1} .
$$

The following theorem states the results to be proven in this section.

TheOREM 1. (a) If $\Omega \subset[0,1] \times(0, A)$ (for some $A>0$ ), then $0 \leqslant u(x, y)$ $\leqslant w_{A}(y)$ for all $(x, y) \in \Omega$.

(b) If $\bar{\Omega} \subset \Omega$, then $\bar{H}-H=\int_{\bar{\Gamma}} \bar{u}(p)\left(\lambda u(p)-\mathbf{D}_{n} u(p)\right)|d p|$.

(c) Assume $\Gamma$ contains no point $p$ at which the normal vector $n(p)$ (directed into

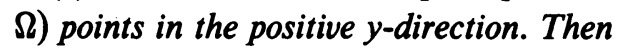

$$
H \geqslant \lambda \lambda^{*}\left(\lambda+\lambda^{*}\right)^{-1}-\lambda^{2} \iint_{\Omega} w_{y}^{2}(y) d x d y .
$$

Here, $w_{y}(y)=\lambda^{*}\left(\lambda+\lambda^{*}+\lambda \lambda^{*} y\right)^{-1}$, as one obtains from equation (1).

(d) Let $\{\Omega\}_{A}$ be the class of regions such that $|\Omega| \leqslant A$ and the condition in part (c) is satisfied. Then $[0,1] \times(0, A)$ is uniquely heat-flow minimizing in $\{\Omega\}_{A}$.

Proof of (a). Direct calculation shows that $\left|\nabla w_{A}(p)\right| \leqslant \lambda w_{A}(p)$ for all $p \in[0,1] \times[0, A]$. Therefore if $v(p):=w_{A}(p)-u(p)$, then $v(p)$ is a harmonic function on $\Omega$ which satisfies the boundary conditions: $D_{n} v=\lambda^{*} v$ on $\Gamma^{*}, \mathbf{D}_{n} v \leqslant \lambda v$ on $\Gamma$, and $\mathbf{D}_{x} v=0$ on $(\{0,1\} \times R) \cap \Omega$. Since $v(p)$ must achieve its minimum value at a point on $\Gamma^{*} \cup \Gamma$ (this follows from the usual maximum principle for the region $\omega$ defined in the Appendix) we conclude that $v(p) \geqslant 0$ in $\Omega$.

Proof of (b). We have $\bar{H}-H=-\int_{\Gamma} \cdot \mathrm{D}_{n} v(p)|d p|$, where $v:=\bar{u}-u$ on 
$\bar{\Omega} . v$ is harmonic in $\bar{\Omega}$ and satisfies the boundary conditions: $\mathbf{D}_{n} v=\lambda^{*} v$ on $\Gamma^{*}, \mathbf{D}_{n} v-\lambda v=\lambda u-\mathbf{D}_{n} u$ on $\bar{\Gamma}$, and $\mathbf{D}_{x} v=0$ on $(\{0,1\} \times R) \cap \bar{\Omega}$. Substitution of the boundary conditions on $\Gamma^{*}$ yields

$$
\begin{aligned}
\bar{H}-H & =-\lambda^{*} \int_{\Gamma^{*}} v=\int_{\Gamma^{*}}\left[v\left(\mathbf{D}_{n} \bar{u}-\lambda^{*} \bar{u}\right)-\bar{u}\left(\mathbf{D}_{n} v-\lambda^{*} v\right)\right] \\
& =\int_{\Gamma^{*}}\left(v \mathbf{D}_{n} \bar{u}-\bar{u} \mathbf{D}_{n} v\right) .
\end{aligned}
$$

Then Green's second identity, applied to the functions $v$ and $\bar{u}$ on $\bar{\Omega}$, yields

$$
\begin{aligned}
\bar{H}-H & =\int_{\Gamma}\left(\bar{u} \mathbf{D}_{n} v-v \mathbf{D}_{n} \bar{u}\right)=\int_{\bar{\Gamma}}\left[\bar{u}\left(\mathbf{D}_{n} v-\lambda v\right)-v\left(\mathbf{D}_{n} \bar{u}-\lambda \bar{u}\right)\right] \\
& =\int_{\bar{\Gamma}} \bar{u}\left(\lambda u-\mathbf{D}_{n} u\right) .
\end{aligned}
$$

The PROOF OF PART (d) (FROM PART (c)). The inequality in (c) reduces to equality for any rectangle $[0,1] \times(0, A)$, as can be shown by direct integration. (From equation (1): the heat flow across $[0,1] \times(0, A)$ is $\lambda \lambda^{*}\left(\lambda+\lambda^{*}+\right.$ $\left.\lambda \lambda^{*} A\right)^{-1}$.) Therefore, the proof that less heat flows across $[0,1] \times(0, A)$ than any other admissible region $\Omega$ with $|\Omega| \leqslant A$ follows from the fact that $w_{y}(y)$ is a positive and strictly monotone decreasing function of $y$ on $[0, \infty)$.

Proof of (c). An open disc with center $p$ and radius $\varepsilon$ is defined by $B_{\varepsilon}(p)=\{q \in[0,1] \times R|| q-p \mid<\varepsilon\}$. Choose a fixed $\varepsilon>0$ so small that $\Omega$ equals the union of all open discs of radius $\varepsilon$ contained in $\Omega$. Let $\alpha^{*}$ $=\max \{y \mid p=(x, y) \in \Gamma\}$. We define the monotone class of regions $\left\{\Omega_{\alpha} \mid 0\right.$ $\left.<\alpha \leqslant \alpha^{*}\right\}$ as follows. If $0<\alpha \leqslant 2 \varepsilon$, then $\Omega_{\alpha}=[0,1] \times(0, \alpha)$. If $2 \varepsilon \leqslant \alpha$ $\leqslant \alpha^{*}$, then $\Omega_{\alpha}$ is the union of all open discs of radius $\varepsilon$ which are contained in $\Omega \cap([0,1] \times(0, \alpha))$. The class of regions $\left\{\Omega_{\alpha} \mid 0<\alpha \leqslant \alpha^{*}\right\}$ thus defined has the following properties. (1) For each $\alpha \in\left(0, \alpha^{*}\right]$, the upper boundary $\Gamma_{\alpha}$ of $\Omega_{\alpha}$ is a simple arc with bounded curvature which is horizontal at its endpoints. (2) For each $\alpha$, the solution $u_{\alpha}(p)$ of our boundary value problem on $\Omega_{\alpha}$ exists. Moreover, $\nabla u_{\alpha}(p)$ has a continuous extension to Closure $\left(\Omega_{\alpha}\right)$ which is in fact Hölder continuous (exponent $\eta$ ) for any $0<\eta<1$. (3) For each $\alpha$, the heat flow $H_{\alpha}$ crossing $\Omega_{\alpha}$ is defined. $H_{\alpha}$ is a continuous function of $\alpha$ on $\left(0, \alpha^{*}\right]$. (Proofs for properties (2) and (3) are outlined in the appendix.) (4) $\Omega_{\alpha} \subset \Omega_{\beta}$ for $0<\alpha \leqslant \beta \leqslant \alpha^{*}, \Omega_{\alpha^{*}}=\Omega$, and $\cup_{0<\alpha<\alpha^{*}} \Gamma_{\alpha}=\Omega \cup \Gamma$.

We now proceed to estimate the left-hand derivative $\mathrm{D}_{\alpha}^{-} H_{\alpha}$ $=\operatorname{limit}_{\delta \rightarrow 0+}\left(\left(H_{\alpha}-H_{\alpha-\delta}\right) / \delta\right)$ at each $\alpha \in\left(0, \alpha^{*}\right]$. For $0<\alpha-\delta<\alpha \leqslant \alpha^{*}$, we have from Theorem $1(\mathrm{~b})$ that

$$
H_{\alpha-\delta}-H_{\alpha}=\int_{\Gamma_{\alpha-\delta}} u_{\alpha-\delta}\left(\lambda u_{\alpha}-\mathbf{D}_{n} u_{\alpha}\right)
$$

It follows from the Hölder continuity of $\nabla u_{\alpha}$ on Closure $\left(\Omega_{\alpha}\right)$ (with exponent $\eta)$ that $\left|\lambda u_{\alpha}-\mathbf{D}_{n} u_{\alpha}\right|=O\left(\delta^{\eta}\right)$ on $\Gamma_{\alpha-\delta}$. Therefore, $\left|u_{\alpha-\delta}-u_{\alpha}\right|=O\left(\delta^{\eta}\right)$ in $\Omega_{\alpha-\delta}$ by the maximum principle, and it follows that 


$$
H_{\alpha-\delta}-H_{\alpha}=\int_{\Gamma_{\alpha-\delta}} u_{\alpha}\left(\lambda u_{\alpha}-\mathrm{D}_{n} u_{\alpha}\right)+O\left(\delta^{2 \eta}\right) .
$$

The divergence theorem on the region $\omega_{\delta}:=\Omega_{\alpha} \mid \Omega_{\alpha-\delta}$ implies that

$$
\iint_{\omega_{\delta}}\left|\nabla u_{\alpha}\right|^{2}=\int_{\Gamma_{\alpha-\delta}} u_{\alpha} \mathrm{D}_{n} u_{\alpha}-\int_{\Gamma_{\alpha}} u_{\alpha} \mathrm{D}_{n} u_{\alpha}
$$

(Here, the normal derivative $D_{n} u_{\alpha}$ is directed into $\Omega_{\alpha}$ on $\Gamma_{\alpha}$ and into $\Omega_{\alpha-8}$ on $\Gamma_{\alpha-\delta}$ as usual.) By substituting equation (4) into equation (3) and applying the condition: $\mathrm{D}_{n} u_{\alpha}=\lambda u_{\alpha}$ on $\Gamma_{\alpha}$, one obtains

$$
H_{\alpha-\delta}-H_{\alpha}=\lambda\left[\int_{\Gamma_{\alpha-\delta}} u_{\alpha}^{2}-\int_{\Gamma_{\alpha}} u_{\alpha}^{2}\right]-\iint_{\omega_{\delta}}\left|\nabla u_{\alpha}\right|^{2}+O\left(\delta^{2 \eta}\right) .
$$

However, if $\gamma_{\alpha}=\Gamma_{\alpha} \cap \Omega$, then

$$
\operatorname{limit}_{\delta \rightarrow 0+} \frac{1}{\delta} \iint_{\omega_{\delta}}\left|\nabla u_{\alpha}\right|^{2}=\int_{\gamma_{\alpha}} \phi\left|\nabla u_{\alpha}\right|^{2}
$$

where $\phi(p)=\operatorname{limit}_{\delta \rightarrow 0+}(1 / \delta) \cdot \operatorname{Distance}\left(p, \Gamma_{\alpha-\delta}\right) \geqslant 0$ for each $p \in \gamma_{\alpha}$. Also,

(7) $\quad \operatorname{limit}_{\delta \rightarrow 0+} \frac{\lambda}{\delta}\left[\int_{\Gamma_{\alpha-\delta}} u_{\alpha}^{2}-\int_{\Gamma_{\alpha}} u_{\alpha}^{2}\right]=\lambda \int_{\gamma_{\alpha}} \phi\left(\mathbf{D}_{n} u_{\alpha}^{2}-\kappa u_{\alpha}^{2}\right)=\int_{\gamma_{\alpha}} \phi\left(2 \lambda^{2}-\lambda \kappa\right) u_{\alpha}^{2}$,

where $\kappa(p)$, which represents the curvature of $\gamma_{\alpha}$ at $p \in \gamma_{\alpha}$, is piecewise constant and assumes only the values 0 and $(1 / \varepsilon)$. In the case where $\frac{1}{2}<\eta<1$, equations (5), (6), and (7) show that $\mathrm{D}_{\alpha}^{-} H_{\alpha}$ exists for each $\alpha \in\left(0, \alpha^{*}\right]$. Since $\left|\nabla u_{\alpha}(p)\right| \geqslant D_{n} u_{\alpha}(p)=\lambda u_{\alpha}(p)$ at each $p \in \gamma_{\alpha}$, we obtain from equations (5), (6), and (7) the estimate

$$
-\mathrm{D}_{\alpha}^{-} H_{\alpha} \leqslant \lambda^{2} \int_{\gamma_{\alpha}} \phi u_{\alpha}^{2}, \quad \alpha \in\left(0, \alpha^{*}\right] .
$$

From the expression for $w_{\alpha}(y)$ in equation (1) and the inequality in Theorem 1(a) we obtain (for $\alpha \in\left[\varepsilon, \alpha^{*}\right]$ ) the inequalities

$$
\begin{aligned}
\mathbf{D}_{\alpha}^{-} \iint_{\Omega_{\alpha}} w_{y}^{2}(y) d x d y & =\int_{\gamma_{\alpha}} \phi(p) w_{y}^{2}(y)|d p| \geqslant w_{\alpha}^{2}(\alpha) \int_{\gamma_{\alpha}} \phi \\
& \geqslant w_{\alpha}^{2}(\alpha-\varepsilon) \int_{\gamma_{\alpha}} \phi-C \varepsilon \geqslant \int_{\gamma_{\alpha}} \phi u_{\alpha}^{2}-C \varepsilon,
\end{aligned}
$$

where $C$ can be chosen uniformly over all $\alpha$ and $\varepsilon$ with $0<\alpha-\varepsilon<\alpha \leqslant \alpha^{*}$. By combining equations (8) and (9), we obtain

$$
\mathrm{D}_{\alpha}^{-} F(\alpha)>0 \text { for } \varepsilon<\alpha \leqslant \alpha^{*},
$$

where

$$
F(\alpha)=H_{\alpha}+\lambda^{2} \iint_{\Omega_{\alpha}} w_{y}^{2}(y) d x d y+\lambda^{2} C \varepsilon \alpha
$$


Since $\Omega_{\alpha}=[0,1] \times(0, \alpha)$ for $\alpha \in(0, \varepsilon)$, it is easily shown that inequality (10) also holds in this case and that limit ${ }_{\alpha \rightarrow 0+} H_{\alpha}=\lambda \lambda^{*}\left(\lambda+\lambda^{*}\right)^{-1}$. Since $F(\alpha)$ is continuous on $\left(0, \alpha^{*}\right]$, it follows essentially by the intermediate value theorem that

$$
F\left(\alpha^{*}\right) \geqslant \operatorname{limit}_{\alpha \rightarrow 0+} F(\alpha)=\lambda^{*}\left(\lambda+\lambda^{*}\right)^{-1}
$$

Therefore

$$
H=H_{\alpha^{*}} \geqslant \lambda \lambda^{*}\left(\lambda+\lambda^{*}\right)^{-1}-\lambda^{2} \iint_{\Omega} w_{y}^{2}(y) d x d y-\lambda^{2} C \varepsilon \alpha^{*} .
$$

The inequality asserted in part (c) now follows from the fact that $\varepsilon>0$ can be chosen arbitrarily small.

3. Doubly-connected regions in $R^{2}$. In this section we assume $\Omega$ is a doublyconnected region whose inner and outer boundary components are, respectively, the unit circle $\Gamma^{*}$ and a closed Jordan curve $\Gamma$ having bounded curvature. The temperature $u(p)$ is a harmonic function in $\Omega$ satisfying the boundary conditions: $\mathrm{D}_{n} u=\lambda u$ on $\Gamma$ and $\mathrm{D}_{n} u=\lambda^{*}(u-1)$ on $\Gamma^{*}$. The rate of heat flow across $\Omega$ is $H=-\int_{\Gamma} \cdot D_{n} u(p)|d p|$.

The solution of our temperature problem on the annulus $R(\alpha)=\{(r, \theta) \mid$ $1<r<\alpha, 0<\theta<2 \pi\}$ (for any $\alpha>1$ ) is given by

$$
w_{\alpha}(r, \theta)=w_{\alpha}(r)=\left(\lambda^{*}+\lambda^{*} \lambda \alpha \operatorname{Ln}(\alpha / r)\right)\left(\alpha \lambda+\lambda^{*} \lambda \alpha \operatorname{Ln}(\alpha)+\lambda^{*}\right)^{-1}
$$

We summarize the results corresponding to Theorem 1 in the present context.

Theorem 2. Assume $\lambda \geqslant 1$ and $\alpha>1$. Then:

(a) If $\Omega \subset R(\alpha)$, then $0 \leqslant u(r, \theta) \leqslant w_{\alpha}(r)$ for each $(r, \theta) \in \Omega$.

(b) If $\Gamma$ contains no point $p$ at which the normal vector $n(p)$ (directed into $\Omega)$ points in the outward radial direction, then

$$
H \geqslant 2 \pi \lambda^{*} \lambda\left(\lambda^{*}+\lambda\right)^{-1}-\lambda \iint_{\Omega}\left(\lambda-\frac{1}{r}\right) w_{r}^{2}(r) r d r d \theta,
$$

where $w_{r}(r)=\lambda^{*}\left(r \lambda+\lambda^{*} \lambda r \operatorname{Ln}(r)+\lambda^{*}\right)^{-1}$.

(c) Let $\{\Omega\}_{\alpha}$ be the class of regions such that $|\Omega| \leqslant \pi\left(\alpha^{2}-1\right)$ and the condition on $\Gamma$ in part (b) is satisfied. Then the annulus $R(\alpha)$ is uniquely heat-flow minimizing in $\{\Omega\}_{\alpha}$.

The proofs are analogous to those in \$2. (Clearly Theorem 1(b) carries over without change.)

4. Remarks concerning a more general problem. Let $\{\Omega\}_{A}$ be the class of doubly-connected regions $\Omega$ with $|\Omega| \leqslant A$, whose inner boundary component is a fixed closed curve $\Gamma^{*}$. If a region $\Omega$ is heat-flow minimizing (subject to our radiation conditions) in $\{\Omega\}_{A}$ and has a sufficiently smooth outer boundary $\Gamma$, 
then a variational procedure shows that the quantity $u(p)\left(\lambda^{2} u(p)-D_{n}^{2} u(p)\right)$ is a constant along $\Gamma$. That a boundary $\Gamma$ satisfying this condition actually exists and is heat-flow minimizing has not been proven. (In the limiting case where $\lambda^{*}=\lambda=\infty$, the necessary condition reduces to: $\mathbf{D}_{n} u$ constant on $\Gamma$. In this case, the heat-flow-minimizing boundary $\Gamma$ exists provided that $\Gamma^{*}$ bounds a starlike region. See Acker [1].) This paper has handled the case where $\lambda^{*}>0, \lambda \geqslant 1$, and $\Gamma^{*}$ is the unit circle.

\section{Appendix.}

LEMMA. In the context of $\$ 2$ :

(a) $\nabla u(p)$ has a continuous extension to Closure $(\Omega)$. In fact $\nabla u(p)$ is Hölder continuous on Closure $(\Omega)$ for any Hölder exponent $0<\eta<1$.

(b) $H_{\alpha}$ is a continuous function of $\alpha$ on $\left(0, \alpha^{*}\right]$.

Proof of part (a). The region $\Omega_{e}=\{(x, y) \mid(x, y) \in \Omega$ or $(-x, y) \in \Omega\}$ maps under $F(z)=\exp (-i \pi z)$ into a doubly-connected region $\omega$ whose inner and outer boundary components $\gamma^{*}$ and $\gamma$ have bounded curvature. The solution $u(p)$ (extended to $\Omega_{e}$ by $u(x, y)=u(-x, y)$ ) maps under $F(z)$ into a harmonic function $v(p)$ in $\omega$ which satisfies the boundary condition

$$
\mathbf{D}_{n} v(p)=\lambda(p) v(p)+f(p) \quad \text { on } \partial \omega=\gamma^{*} \cup \gamma .
$$

Here, $\lambda(p)=(\lambda / \pi|p|), f(p)=\lambda$ on $\gamma^{*}$, and $f(p)=0$ on $\gamma$. Now $v(p)$ can be expressed in the form

$$
\nu(p)=\int_{\partial \omega} \psi(q) \operatorname{Ln}(|q-p|)|d q|,
$$

where $\psi(p)$ (defined on $\partial \omega$ ) is a solution in $H(1)$ (i.e., Hölder continuous for any exponent $0<\eta<1)$ of the integral equation

$$
\begin{array}{r}
\pi \psi(p)-\int_{\partial \omega} \psi(q)\left[\frac{(q-p) \cdot n(p)}{|q-p|^{2}}+\lambda(p) \operatorname{Ln}(|q-p|)\right]|d q|= \\
\quad f(p), \\
\quad p \in \partial \omega .
\end{array}
$$

(See [6, XVIII, §3].) One can show using well-known integral formulas for

$$
\frac{\partial v(p)}{\partial n(p)}=\operatorname{limit}_{q \rightarrow p} \frac{\partial v(q)}{\partial n(p)} \text { and } \frac{\partial v(p)}{\partial T(p)}=\operatorname{limit}_{q \rightarrow p} \frac{\partial v(q)}{\partial T(p)}
$$

(where $p \in \partial \omega, q \in \omega$, and $T(p)$ is a unit tangent to $\partial \omega$ at $p$ ) that these functions are in $H(1)$ on $\partial \omega$. (See [5, 2, §13] and [4, \$29].) Thus, the function $G(z)=\mathbf{D}_{x} v(z)-i \mathbf{D}_{y} v(z)$ (analytic in $\omega$ ) has a continuous extension to $\partial \omega$ which is in $H(1)$ on $\partial \omega$. Thus, $G(z)$ (or $\nabla v(p))$ is in $H(1)$ on $\omega \cup \partial \omega$ by [5, 2, 822].

Proof of PART (b). For each $0<\beta \leqslant \alpha^{*}$, let $\omega_{\beta}$ correspond to $\Omega_{\beta}$ as in part (a), and let equation (15 $)$ refer to equation (15) when $\omega=\omega_{\beta}$. If $\beta \rightarrow \alpha$, 
one can show by considering equation $(15 \beta)$ as a perturbation of equation $(15 \alpha)$ that $u_{\beta}(p) \rightarrow u_{\alpha}(p)$ (and therefore that $\nabla u_{\beta}(p) \rightarrow \nabla u_{\alpha}(p)$ ) uniformly on any compact subset of $\Omega_{\alpha}$. The result follows from this.

\section{REFERENCES}

1. A. Acker, Heat flow inequalities with applications to heat flow optimization problems, SIAM J. Math. Anal. (to appear).

2. T. Carleman, Über ein Minimalproblem der mathematischen Physik, Math. Z. 1 (1918), $208-212$.

3. G. M. Golusin, Geometrische Functiontheorie, VEB Deutscher Verlag, Berlin, 1957.

4. J. Horn, Partielle Differentialgleichungen, de Gruyter, Berlin, 1949.

5. N. I. Mushelišvili, Singular integral equations, Noordhofi, Groningen, 1953.

6. W. Pogorzelski, Integral equations and their applications, Vol. I, PWN, Warsaw, 1966.

7. G. Szegö, Ober einige Extremalaufgaben der Potentialtheorie, Math. Z. 31 (1930), 583-593.

Mathematischis Institut I, Universttät Karlskuhie (TH), Fbderal Rbpubuc of GbrunNy 